\title{
A metric to assist the product families architecture definition
}

\author{
José Nilton Martini", Fernando Antônio Forcellinib \\ Universidade do Estado de Santa Catarina \\ bUniversidade Federal de Santa Catarina \\ e-mails: jose.martini@udesc.br; fernando.forcellini@ufsc.br
}

\begin{abstract}
It is difficult to define products architecture for two main reasons: the high number of architecture alternatives and the lack of an evaluation criteria to these options. This article addresses the alternative architectures evaluation issue using a cost estimate approach. The purpose of this study is to describe a metric that assists the product architecture definition. The metric showed here is built over the relative estimates of the developing and assembly costs and was created as a tool to define a product family architecture; It uses the products cost estimates and functional descriptions. In addition to describing the metric that helps to define the product architecture, this article also illustrates its application to a family of four products sharing the same functional model. Finally, the metric presented is analyzed, as well as the potential to its use with computational algorithms to deal with complex problems.
\end{abstract}

Keywords: product architecture, evaluation metric, product family.

\section{Introduction}

The most important aspect of product architecture is the way that functions are mapped to physical components. At the beginning of the process of developing new products, when only the functional models are available, the definition of the architecture is generally confined to the choices of the functional groups that are developed in conjunction as components. Of the possible mapping options among the product functions and components, two stand out. One is the mapping of all the product functions in only one component. The other is the so-called one-for-one mapping among the product functions and components. In the first case, it is said that the product has a totally integral architecture. In the second, it is said that the product has a totally modular architecture (as long as the interfaces between the components are uncoupled) (ULRICH, 1995). Figure 1 exemplifies these architectures: (A) illustrates a product that executes its three functions with only one component $-\mathrm{a}$ totally integrated architecture and (C) illustrates a product whose functions are executed by specific components - a totally modular architecture (in the two examples the question of interfaces was not considered).

Among the mappings that define the totally integral and modular architectures there are countless options of component-function maps. These alternatives are called, in this text, modular focus architectures and are characterized by components that completely execute one or more functions (the functions are not executed in a way that is distributed among the components). Figure 1B illustrates an alternative architecture of modular focus for a product of three functions that are executed by two components - one component totally executes two functions and the second component executes the other function. The completely modular and integral architectures can be seen as particular cases of the set of alternative architectures with a modular focus (from the perspective of component-function mapping).

Two architectures whose functions are executed, to a certain degree, in a distributed manner among the components, can also be defined. The first of these is called, in this article, architecture with a high degree of integration. This architecture is defined by a complex map that is ambiguous in terms of the product functions and components. The second is called (in this article) hybrid architecture and is characterized by the fact that some components completely execute one or more functions, while other components execute portions of one or more functions. Figure 2 illustrates these architectures: (D) is an example of an architecture with a high degree of integration (the three product functions are executed partially by each one of the three components), and (E) illustrates a hybrid architecture, two functions of the product are executed in a distributed form among the components and a function is executed by a specific component.

The adoption of the modular focus or totally modular architectures for the products can be advantageous, for many reasons, for companies and consumers. According 


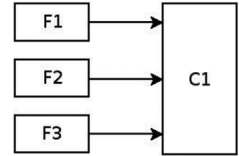

(A)

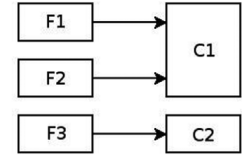

(B)

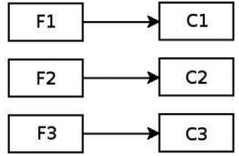

(C)
Figure 1. Totally integral (A), modular focus (B) and totally modular (C) product architectures.

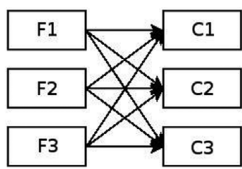

(D)

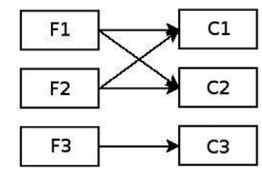

(E)
Figure 2. Architecture (D) has a high degree of integration and $(\mathrm{E})$ is hybrid.

to Erens and Verhulst (1997), these architectures generate stability in the interfaces, reduce communication between the developers of the different components, define the communality and the variety of the components and facilitate the reuse and updating of the product components. For Ulrich (1995), the adoption of these architectures makes it easier to alter the product during its life, motivated by the evolution of the product, the addition of its functionalities, adaptation, component wear, consumption and flexibility of use. According to Pahl et al. (2005), consumers perceive a series of advantages in products with modular architectures in comparison with traditional products, including: shorter delivery periods, better possibilities for substitution and repair, better service of replacement parts, later modifications and extensions of the function within the limits of the spectrum of variations and the practical elimination of chances for failure, given the maturing of the layout.

Despite the advantages derived from the adoption of a modular focus architecture for the products, its definition is not simple. The first difficulty is the high number of alternatives for architectures with possible modular focuses. The quantity of alternative modular focus architectures for a product can be calculated by the Bell numbers, which provide the number of not vacant disconnected partitions that can be obtained from a set with $n$ elements; the Bell number is obtained from the sum of the second species Stirling numbers (which are calculated recursively) (DICKAU, 2006). One can have an idea of the number of alternatives of modular focus architecture by calculating the Bell number for a product with 5 and 20 functions. For 5 functions the number of alternative modular focus architectures for the product is 52, while for 20 functions it is 5.132E13, or that is, for a product of 20 functions it is impossible (considering the processing capacity of current computers and of a reasonable time period) to evaluate all the alternatives.

The second problem in the definition of a modular focus architecture for the products is the choice of the functional groups that will be developed as components (modularization process). According to Yang, Beiter and Ishii (2005) and Hölttä-Otto (2005), three methods for the definition of these groupings stand out in scholarly journals. The first of these, proposed by Stone, Wood and Crawford (1998), is based on the Structure of Functions of the product (product model described by Pahl et al., 2005) and in the application of three flow heuristics proposed by the authors (heuristics: of the dominant flow, branched flow and of transformation). Studies such as that of Dahmus, Gonzalez-Zugasti and Otto (2001) utilized the heuristics proposed by Stone, Wood and Crawford (1998) for the proposal of an approach to assist the definition of that which are, or could be, the common or shared modules of a product family. The second modularization method, called Design Structure Matrix (DSM), uses the representation of the product of the same name for the grouping of the elements (in the case of functions) - this method seeks to reduce the interactions between the groupings formed (BROWNING, 2001). Huang and Kusiak (1998) propose an alternative for the modularization of the products using the DSM proposal. The third popular method for the definition of the functional groupings is called Modular Function Deployment (MFD). This method, proposed by Erixon (1996) relates to the functions of the product with a series of modularization guidelines. The product modules are defined after the filling in of a modularization tool known as the Modular Indication Matrix (MIM). Despite the relative ease of application of the concepts proposed by these methods, it is not possible to know, even relatively, the quality of the architecture option suggested by each one of them.

The metric described in this article is the result of the authors' search for an evaluative approach related to the various options for architectures for product families. This metric, shown in the next sections, seeks to estimate the relative development and assembly costs of the modular focus architecture alternatives for the products. The application of this metric can assist the definition of a modular focus architecture for a product family (or that is a modular focus architecture seeking the generation of the diversity of the supply). The hypotheses and the simplified evaluation model of the architecture options are presented in the next section. Then the model is detailed and its application is illustrated by an example that is developed in parallel to the presentation of the metric. The final section of the article is reserved for comments about the application of the metric proposed and its use in conjunction 
with computational approaches for the definition of the architecture in more complex problems.

\section{Metric based on the evaluation costs of the architecture options}

This section seeks to establish the bases of the cost metric for the relative evaluation of the various architectural alternatives for a product family. The definition of the modular focus architecture specific to a particular product family can be made by choosing the option that has the lowest estimated development and assembly costs using the proposed metric.

The metric based on the evaluation costs of the alternative architectures is composed of two parts. The first estimates the relative costs of a certain architectural alternative for the product family, considering the development costs of its functions. This portion of the costs is derived from the investments realized before the initiation of production and is reflected as part of the costs of the products. The second part involves the cost estimate after the initiation of production and is directly linked to the costs of assembling the products. This part is calculated considering the cost of the interface between the modules (called in this article Assembly Cost I) and the potential reduction of the manufacturing and assembly costs for a certain grouping of functions (module), called in this article assembly cost II) The metric proposed to assist defining the architecture of a product family recognizes that:

- the development costs of a group of functions are equivalent to the sum of the development costs of its individual functions (which can be estimated in a relative manner);

- The costs for the interface between the functions are equal to the assembly costs of the products (and can, therefore, be estimated in a relative form);

- The potential for integration of the functions of a module can be used to estimate the reduction of the production and assembly costs of a functional grouping; and

- The number of functions and its interactions defines the potential for integration of a module.

In a preliminary form, an equation can be written to calculate the cost estimate of a given modular focus architecture option for the product family adding the two cost portions mentioned (development and assembly). Since the nature of the development costs is different from the nature of the assembly costs, it is necessary to establish their weights in the composition of the estimate of the total cost. Equation 1 illustrates the proposed calculation.

$$
C_{E}^{i}=\alpha * C_{D}^{i}+\beta *\left(C_{M-I}^{i}+C_{M-I I}^{i}\right)
$$

In Equation 1, $C_{E}^{I}$ is the estimate of the total costs of the architecture $i$ for the product family, $C_{D}^{I}$ is the development cost of the architecture $i, C_{M-I}^{I}$ is the assembly costs among the modules for architecture $i$, and $C_{M-I I}^{I}$ is the intra-modules assembly cost also for architecture $i$. Also in Equation (1), $\alpha$ is the coefficient that represents the cost element related to the investments realized during the development of the functions of the product family and $\beta$ is the coefficient that represents the part of the costs related to the assembly. The calculation procedures proposed for each of the parts of cost estimate are detailed in the coming sections of the article.

\subsection{Cost estimate for development of the architecture options}

This section proposes a procedure for calculating the costs related to the choice of an architectural alternative for a product family from the perspective of allocation of the functions to the components (or modules) of the products component $C_{D}^{I}$ of Equation 1. To estimate development costs it is necessary to know (or estimate) the costs related to the development of the functions of the product family and the functional descriptions of the products. To help understand the proposal for calculating the estimated development costs consider a family of four products (p1, p2, p3 and p4) that each realize five functions (f1, $\mathbf{f} \mathbf{2}, \mathbf{f 3}, \mathbf{f} \mathbf{4}$ and $\mathbf{f 5}$, each function can present a specific variation for a particular product, for example a performance variation). Table 1 illustrates the description of the four products of this family in the functional space - the numbers listed correspond to the variation of a specific function needed for the formation of each product and Table 2 illustrates an estimate of the

Table 1. Example of the functional description of the products of a family.

\begin{tabular}{|c|c|c|c|c|c|}
\hline \multirow{2}{*}{ Products } & \multicolumn{5}{|c|}{ Functional description of the products } \\
\cline { 2 - 6 } & $\mathrm{f} 1$ & $\mathrm{f} 2$ & $\mathrm{f} 3$ & $\mathrm{f} 4$ & $\mathrm{f} 5$ \\
\hline $\mathrm{p} 1$ & 1 & 1 & 1 & 2 & 1 \\
\hline $\mathrm{p} 2$ & 1 & 1 & 1 & 1 & 2 \\
\hline $\mathrm{p} 3$ & 1 & 3 & 1 & 2 & 1 \\
\hline $\mathrm{p} 4$ & 1 & 2 & 1 & 1 & 3 \\
\hline
\end{tabular}

Table 2. Example of the estimate of the relative cost for development of the functions.

\begin{tabular}{|c|c|c|c|c|c|}
\hline \multirow{2}{*}{ Variations } & \multicolumn{5}{|c|}{ Estimate of the relative costs for development of the } \\
& \multicolumn{7}{|c|}{ functions } \\
\cline { 2 - 6 } & $\mathrm{f} 1$ & $\mathrm{f} 2$ & $\mathrm{f} 3$ & $\mathrm{f} 4$ & $\mathrm{f} 5$ \\
\hline 1 & 5 & 3 & 4 & 1 & 5 \\
\hline 2 & - & 4 & - & 2 & 6 \\
\hline 3 & - & 5 & - & - & 7 \\
\hline
\end{tabular}


investments related to the development of each one of the variations of five functions of the product family.

The development cost of a particular architecture is obtained by adding, for all of its modules, the relative development cost of its functions. Equation 2 illustrates this calculation procedure. The relative development cost, Equation 3, is obtained by dividing the result of the application of Equation 2 by the development cost of the products according to an integral architecture, which is always the highest possible development cost.

$$
\begin{aligned}
& C_{A D}^{k}=\sum_{i=1}^{N_{M}} \sum_{j=1}^{N_{V i}} c d_{i j}^{k} \\
& C_{D}^{k}=\frac{C_{A D}^{k}}{C_{A D}^{I}}
\end{aligned}
$$

In Equation 2, $C^{K}{ }_{A D}$ is the absolute estimated development cost of the architecture $k, c d^{K}{ }_{i j}$ is the estimated development cost of the variation $j$ of the module $i, N_{V i}$ are the variations of architecture module $i$ defined by $N_{M}$ modules. In Equation $3 C^{K}{ }_{D}$ is the relative development cost of architecture $k$ and $C^{I}{ }_{A D}$ is the absolute development cost of an integral architecture for the products.

To illustrate the use of the equations presented, consider the data of Tables 1 and 2. Also consider a particular modular focus architecture for the product family that defines a function-component allocation map as follows: $\{\{\mathrm{f} 1 \mathrm{f} 2\}$ $+\{\mathrm{f3} \mathrm{f} 4 \mathrm{f} 5\}\}$, that is, functions 1 and 2 define one module and functions 3, 4 and 5 define another module.

The estimated development cost for the first variation of a module composed of functions 1 and 2 is $8(5+3$, according to Table 2); although, to form all the products of the family, three variations of this module should be developed: one variation for products $1 \& 2$, one variation for product 3 and one variation for product 4 . The estimated development cost of the first variation of the module composed of functions 3,4 and 5 is $11(4+2+5$, according to Table 2$)$. This module should also be developed in 3 variations to generate the desired product family: one variation for products 1 and 3 , one variation for product 2 and one variation for product 4 . The development costs of the variations of these modules are compiled in Table 3.

The application of Equation 2 to the data of Table 3 results in a development cost for the illustrated architecture $(\{\mathrm{f} 1 \mathrm{f} 2\}+\{\mathrm{f} 3 \mathrm{f} 4 \mathrm{f} 5\})$ of 61 . For the use of this calculation in the expression of the total cost, the development cost should be normalized. The normalization is conducted by dividing the development cost of a particular architecture by the development cost of an integral architecture, Equation (3). Using the data from this example, the development cost of the product family following an integral architecture is 80 . Therefore, the normalized development cost of this particular architecture $(\{\mathrm{f} 1 \mathrm{f} 2\}+\{\mathrm{f3} \mathrm{f} 4 \mathrm{f} 5\})$ is 0.7625 .

As seen, the architecture illustrated is only one of the 52 possible architectural options (from the perspective of function-component allocation) for products defined by 5 functions. In order to identify the estimated relative development cost of the other architectural options, the procedure for calculation described should be repeated for the remaining 51 function-component allocations. With the result of these calculations, a graph can be sketched that illustrates the variation of relative development costs for the product family, proposed in this work, for each architectural alternative - Figure 3. In the Figure, the first architectural alternative (on the left) is integral, or that is, for the example in question, the four products are developed in an individualized manner, not considering their common functions. The last architectural alternative (farther to the right in the figure) corresponds to a totally modular architecture, or that is, the functional commonalities between the members of the product family are explored.

\subsection{Estimated assembly cost of the product family}

After estimating the development costs of the product family for the architectural options, this and the next section will present a proposal for estimating assembly

Table 3. Estimated development costs of the modules.

\begin{tabular}{|c|c|c|}
\hline Module & Variation & $\mathrm{cd}$ \\
\hline \multirow{3}{*}{$\mathrm{f} 1 \mathrm{f} 2$} & 1 & 8 \\
\cline { 2 - 3 } & 2 & 10 \\
\cline { 2 - 3 } & 3 & 9 \\
\hline \multirow{3}{*}{$\mathrm{f} \mathrm{f} 4 \mathrm{f5}$} & 1 & 11 \\
\cline { 2 - 3 } & 2 & 11 \\
\cline { 2 - 3 } & 3 & 12 \\
\hline
\end{tabular}

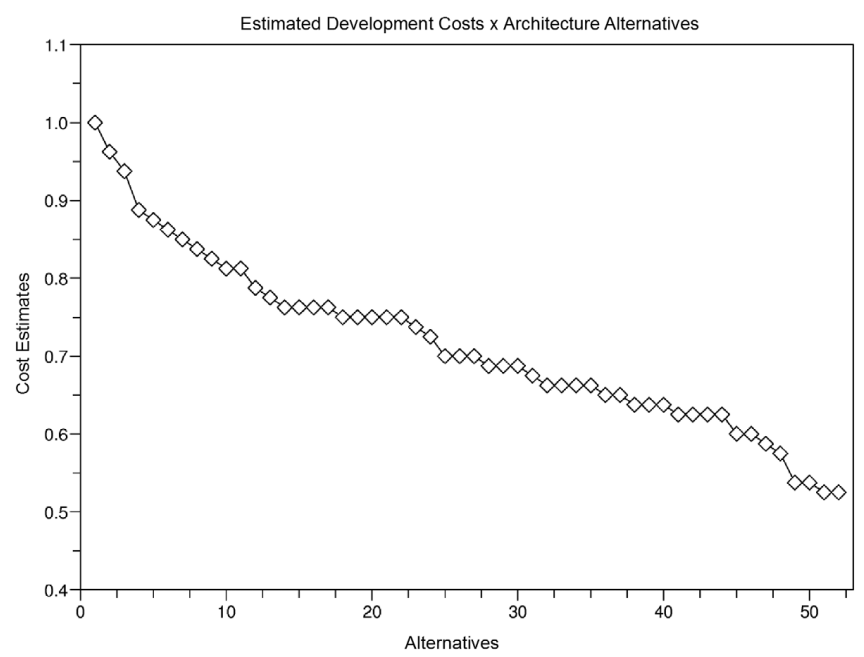

Figure 3. Estimated development costs of the architectural options. 
costs. This section is dedicated to formalizing an estimate of the assembly costs among modules of a product family considering an architectural option with a particular modular focus.

The estimated assembly costs among modules depends on the number of interfaces between the modules of the particular architectural option, the types of interfaces and the estimated assembly costs for each type of interface. This information, with the exception of the estimated assembly costs, can be obtained from the functional models of the product (for example DSM, Structure of Functions, etc.). This proposal assumes that the relative assembly costs for each type of interface can be estimated.

For a particular architecture, the number of interfaces between modules can be obtained, for example, by inspecting the model of the functional interaction of the products. The assembly cost is calculated by multiplying the number of each type of interface between the various product modules by the (estimated) relative assembly cost and by the production volume. Equation 4 illustrates the proposed calculation.. To obtain the relative amount of this cost, Equation 5, the result of the calculation of Equation 4 is divided by the assembly cost of a totally modular architectural alternative, which results in the largest possible assembly cost for the products.

$$
\begin{aligned}
C_{A M-I}^{k} & =\sum_{i=1}^{N_{P}} \sum_{j=1}^{N_{M i}} V P_{i}\left(N I E_{i j}^{m} c m_{m}+N I E_{i j}^{e} c m_{e}+N I E_{i j}^{s} c m_{s}\right) \\
C_{M-I}^{k} & =\frac{C_{A M-I}^{k}}{C_{A M-I}^{M}}
\end{aligned}
$$

In Equation 4, $C^{K}{ }_{A M-I}$ is the absolute assembly cost among modules of architecture- $k$ of the product family, $N_{P}$ is the number of modules of product $i, V P_{i}$ is the volume of assembly of product $i, N I E^{M}{ }_{i j}$ is the number of interfaces of material of module $j$ of product $i$ (the superscripts $e$ and $s$ indicate the number of interfaces of energy and sign, respectively), $\mathrm{cm}_{m}$ is the relative assembly cost for the interfaces of flow of material (the subscripts $e$ and $s$ respectively indicate the relative assembly costs for the interfaces with flow of energy and sign). In Equation 5, $C^{K}{ }_{M-I}$ is the relative assembly cost among modules with architecture $k$ and $C^{M}{ }_{A M-I}$ is the assembly cost of the products following a totally modular architecture.

For purposes of illustration of the application of Equations 4 and 5, consider the set of products and the particular architecture for the family proposed in the previous section. Consider that the functional model (from the perspective of the system) of Figure 4 for the products of five functions (f1, f2, $\mathbf{3} \mathbf{3}, \mathbf{f} \mathbf{4}$ e f5; $\mathbf{f s}$ is the frontier of the system) would be representative of the products of the family. The functions of the products and the functional interactions of the material (heavy continuous line), energy (continuous line) and sign (dashed line) are illustrated in this figure. Also consider that the relative assembly costs for each type of interface are estimated according to the data in Table 4.

The number of each one of the types of interfaces between the modules can be obtained by inspection of the two modules suggested in the example for calculating the cost estimate for this section - this information is summarized in Table 5.

The application of Equation 4 with the data from Tables 5 and 6, considering production volumes of 100 units of product 1, 80 of product 2, 60 of product 3 , and 30 of product 4 , result in an assembly cost between modules for the volume of production and for this particular architecture of 6750 . For a totally modular architecture, this assembly cost is 10530, the highest among all the architectural options of a modular focus, used for normalization. The estimated normalized cost Equation 5, for the architectural option suggested, therefore, is 0.6410 (14th option in the graph of Figure 5 that illustrates the variation of these costs with the architectural alternatives). It can be seen that the lowest

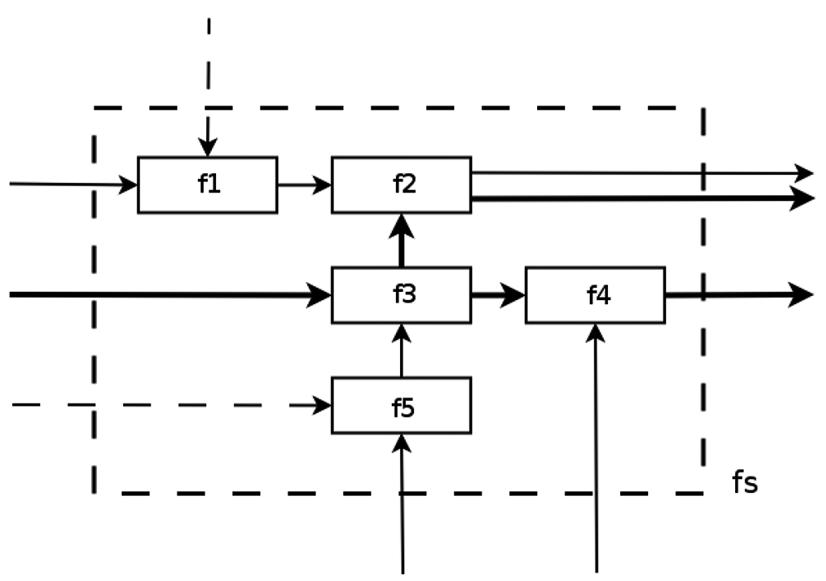

Figure 4. Functional model for the products of the family.

Table 4. Estimate of the relative assembly costs per type of interface.

\begin{tabular}{|l|c|c|c|}
\hline \multirow{2}{*}{$\begin{array}{l}\text { Relative assembly } \\
\text { cost(estimated) }\end{array}$} & \multicolumn{3}{|c|}{ Type of interface } \\
\cline { 2 - 4 } & Material & Energy & Sign \\
\cline { 2 - 4 } & 3 & 2 & 1 \\
\hline
\end{tabular}

Table 5. Number of interfaces between modules for the example suggested.

\begin{tabular}{|c|c|c|c|}
\hline \multirow{2}{*}{ Modules } & \multicolumn{3}{|c|}{ Interfaces between modules } \\
\cline { 2 - 4 } & Material & Energy & Sign \\
\hline$\{\mathrm{f} 1 \mathrm{f} 2\}$ & 2 & 2 & 1 \\
\hline$\{\mathrm{f} 3 \mathrm{f} 4 \mathrm{f} 5\}$ & 3 & 2 & 1 \\
\hline
\end{tabular}


Table 6. Relationship between the modules, their internal interfaces and the non-related functions.

\begin{tabular}{|c|c|c|c|c|c|c|}
\hline \multirow{2}{*}{ Modules } & \multicolumn{3}{|c|}{ Intra modular interfaces } & \multicolumn{3}{c|}{ Non-related functions } \\
\cline { 2 - 7 } & Material & Energy & Sign & Material & Energy & Sign \\
\hline$\{\mathrm{f} 1 \mathrm{f} 2\}$ & - & 2 & - & - & 2 & - \\
\hline f3 f4 f5 & 2 & 2 & - & 2 & - \\
\hline
\end{tabular}

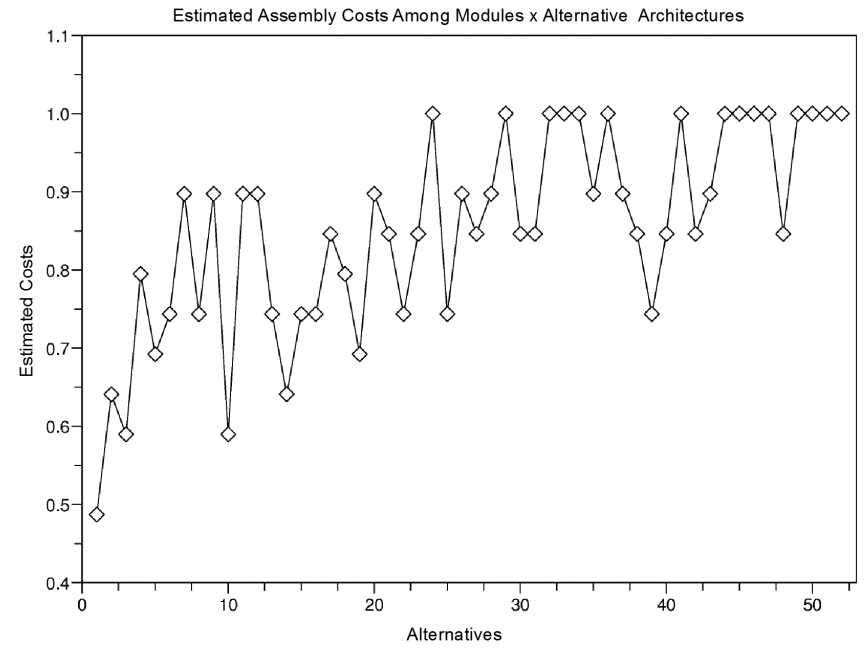

Figure 5. Estimated assembly costs among modules of alternative architectures.

estimated assembly cost corresponds to the totally integral architecture (first mark to the left in the graph) and the highest estimated assembly cost corresponds to the totally modular architecture (last mark on the right of the graph). It can also be seen that the many architectural options of a modular focus have the highest assembly cost among the modules, this is true for the architectures that define modules with functions with no relationship between each other.

\subsection{Potential for integration of the modules}

Until now, the assembly costs of a product formed by a distinct set of modules has depended on the number of interfaces between the modules and the estimated assembly costs. Imagine, initially, that whatever functions that define a particular module can be integrated. With the presumed integration, the interfaces between the function of a single module are not considered, eliminating these assembly costs.

Nevertheless, the integration cannot be assumed as certain for any functional grouping, revealing the need for a measure of the potential of integration of the functions of a particular module. In later stages of product development, when information about the components is already available, the potential for integration of the parts can be estimated by an analysis of the similarities of the process and material (for example). In the initial stages of product development, however, this information is not available, forcing the definition of an estimate of the potential for integration of a module using only systemic information about the product.

In this section, a method is proposed to estimate that part of the cost of (production and) assembly that can, eventually, be eliminated with the integration of the functions of a module. In this proposal, only the functional models of the products are considered to be known, which represent the exchanges of material, energy and sign between the functions.

The definition of the potential for integration proposed in the article is composed of two components. The first of these is the ratio between the number of interactions and the number of functions of a functional grouping (module). Assume that the integration potential of the functions of the grouping is directly related to this component; the larger the ratio, the greater the possibility of functional integration (this ratio can vary between 0 and $\sim 1$ ). The second component considered important to define the integration potential is the ratio between the number of functions that interact with the other functions of the functional grouping and the total number of functions of the grouping. Also in this case, the larger the ratio (which varies between 0 and 1), the more likely the functional grouping is to integrate. Therefore, when defining the calculation of the integration potential of the proposal consider: the number of functions of the grouping; the number of interactions of material, energy and sign between the functions of the grouping; and the number of functions that interact with the other functions of the grouping.

To calculate the estimate of the potential of integration of a module, the two ratios are multiplied. This proposal for calculating the integration potential of a module can be placed in algebraic form, as follows (the number 2 that appears in the denominator of the equation for estimating the integration potential is used because the interactions between the functions are counted in pairs):

$$
P_{M}^{m}=\frac{N_{M}^{m} N_{M}^{f m}}{2 N_{M}^{f 2}}
$$

In Equation 6, $P^{M}{ }_{M}$ is the integration potential of the functions of module $M$ for the elimination of the interfaces of the flow of material, $N^{F}{ }_{M}$ is the number of functions of the module, $N^{M}{ }_{M}$ is the number of interfaces of the flow of material of the module, and $N^{F M}{ }_{M}$ is the number of functions that interact with the other functions of the grouping, from the perspective of the flows of materials. Equations 
similar to (6) can be written for the integration potentials of the functions of a grouping from the perspective of the interactions of energy and sign.

In the formulation proposed for the potential of functional integration, the reduced assembly cost of a module will be proportional to the integration potential. The integration potential of the functions of a grouping can be seen as the possibility for reduction of costs of (production and) assembly of a module. A potential equal to the unit means that there is a potential for integration of all the grouping functions, therefore, the assembly cost of this module can be considered to be equal to zero. On the other hand, an integration potential equal to zero results in an assembly cost of the module equal to the sum of the interface costs between the functions of the module. Therefore, for module $M$, the intramodular assembly cost is obtained by the sum of the difference between these potentials and the unit, multiplied by the respective interface costs (the same used in the estimate of the assembly costs) and by the number of intra module interfaces. Finally, the intra module assembly costs of an alternative architecture can be estimated by adding these costs to each one of the modules of the products of the family. Equation 7 illustrates this calculation procedure. As before, this cost estimate can be normalized considering the maximum possible assembly cost for a totally modular architecture, Equation 8.

$$
\begin{aligned}
& C_{A M-I I}^{k}=\sum_{i=1}^{N_{P}} \sum_{j=1}^{N_{M i}} V P_{i}\left(\begin{array}{l}
\left(\left(1-P_{i j}^{m}\right) N I I_{i j}^{m} c m_{m}\right)+ \\
\left(\left(1-P_{i j}^{e}\right) N I I_{i j}^{m} c m_{e}\right)+ \\
\left(\left(1-P_{i j}^{s}\right) N I I_{i j}^{s} c m_{s}\right)
\end{array}\right) \\
& C_{M-I I}^{k}=\frac{C_{A M-I I}^{k}}{C_{A M-I}^{M}}
\end{aligned}
$$

In Equation 7, $C^{K}{ }_{A M-I I}$ is the absolute assembly cost of the internal function of the modules of architecture alternatives $k, N_{P}$ is the number of products of the family, $N_{M i}$ is the number of modules of product $i$ of the family, $V P_{i}$ is the assembly volume of the product $i, P^{*}$ is the integration potential of module $j$ of product $i, N I I^{*}{ }^{*}$ ij the number of internal interfaces to module $j$ of product $i$, and $\mathrm{cm}^{*}$ are the estimated assembly costs (the symbol ' $*$ ' can be: $m$ for material, $e$ for energy, and $s$ for sign). $C^{K}{ }_{M-I I}$ is the relative assembly cost of the intramodular assembly of architecture $k$ and $C^{M}{ }_{A M-I}$ is the assembly cost of the products adopting a totally modular architecture.

To illustrate the use of Equations 7 and 8, consider, once again, the alternative architecture already used as an example $(\{\mathrm{fl} \mathrm{f} 2\}+\{\mathrm{f3} \mathrm{f} 4 \mathrm{f} 5\})$. Inspecting the functional model of the products of the family (Figure 4), the number of interfaces in each module can be obtained, as well as the number of functions with no relation to the others (or to each module); this information is shown in Table 6.

The integration potential of the two modules of this specific architecture, using Equations 7 and 8 and the data from Tables 3 and 7, is illustrated in Table 7. Remembering that the potential for integration of the modules is equal for all the products of the family, given that the products share the same functional model.

By applying Equation 7 to the data presented in Tables 6 and 7, the intramodular assembly cost for the products for the proposed architecture is found in Equation (9):

$$
\begin{gathered}
2640=(100+80+60+30) *\left((1-0.5)^{*}\right. \\
(2 * 2)+(1-0.222) *(2 * 3)+(1-0.222) *(2 * 2))
\end{gathered}
$$

Normalizing this value (considering the assembly cost of 10530 for a totally modular architecture) we find the relative estimated intramodular assembly cost of 0.2507 . Figure 6 illustrates these costs (undergone pouring lozenges) together with the assembly costs between modules (solid lozenges) in the graph for all the architectural alternatives for the family of products (the 14th mark in this graph represents the architecture of the example).

The sum of the assembly costs between modules and intra modules results in the total relative assembly costs of the alternative architectures, Figure 7 presents these costs.

Table 7. Integration potential of the modules.

\begin{tabular}{|c|c|c|c|}
\hline \multirow{2}{*}{ Modules } & \multicolumn{3}{|c|}{ Integration potential } \\
\cline { 2 - 4 } & Material & Energy & Sign \\
\hline$\{\mathrm{f} 1 \mathrm{f} 2\}$ & 0.000 & 0.500 & 0.000 \\
\hline$\{\mathrm{f} 3 \mathrm{f} 4 \mathrm{f} 5\}$ & 0.222 & 0.222 & 0.000 \\
\hline
\end{tabular}

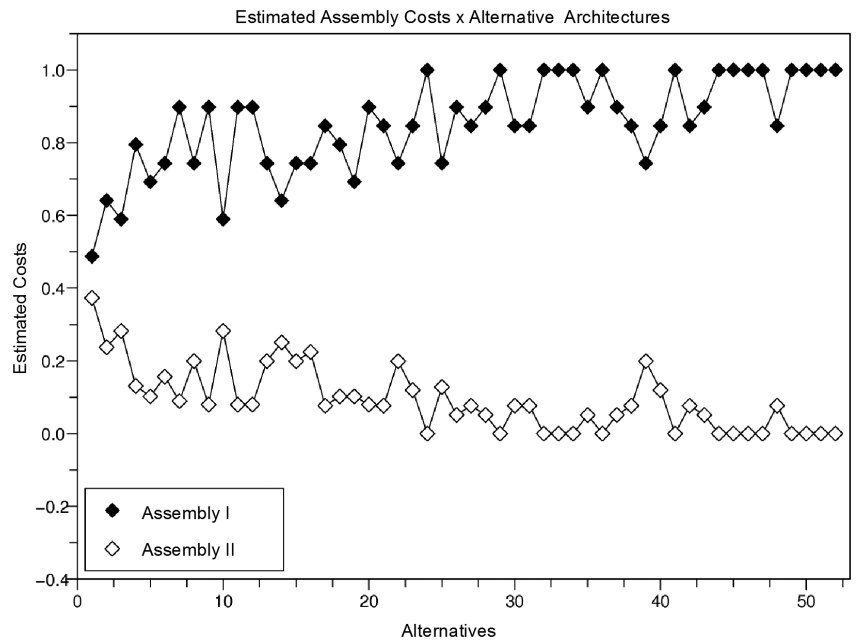

Figure 6. Estimated assembly costs between and intra modules for the alternatives. architectures. 


\subsection{Defining the architecture by applying the proposed cost metric}

After calculating the estimated development and assembly costs, the architecture for the product family can be chosen. It is recognized that the best architecture is that which results in the lowest estimated cost for the family of products. First, however, the values for coefficients $\alpha$ and $\beta$ must be chosen, These represent the cost structure of the company for the development and assembly of the products.

Figure 8 presents the curves of the total cost estimated for various combinations of $\alpha$ and $\beta$ for the data from the example shown in the article. When $\alpha$ is equal to the unit and $\beta$ is equal to zero, we have the curve defined by the hollow diamonds (in this case only the development costs are important). When $\alpha$ is zero and $\beta$ is equal to the unit, we have the curve defined by the solid diamonds ( in this case only the assembly costs are important), The curves defined between these limits depend on the choice of coefficients $\alpha$ and $\beta$. Figure 8 used four pairs for these coefficients $0.2-0.8$; $0.4-0.6$; 0.6-0.4; e $0.8-0.2$ (for $\alpha$ and $\beta$, respectively).

The choice of the coefficients depends, as mentioned, on the company cost structure, but generically one can assume values greater than $\alpha$ (in relation to $\beta$ ) for cases of families with many products and low production volume and higher values of $\beta$ (in relation to $\alpha$ ) for cases of families with few products and a large production volume. Assuming, in this example, the application of the metric, $\alpha$ equal to 0.3 and $\beta$ equal to 0.7 , the best alternative architecture is that which defines the following functional groupings $\{\mathrm{f} 1\}+\{\mathrm{f} 2 \mathrm{f3} \mathrm{f} 4\}+\{\mathrm{f5}\}$ (19th architecture option in the graph of Figure 8).

The functional groupings (modules) suggested after the application of the cost metric are illustrated in Figure 9. The module that executes function 1 is unique for the four products of the family. The module that executes functions 2, 3 and 4 require 4 variations to attend the four products of the family (the three functions of this grouping exchange materials among each other, suggesting the potential for integration and consequently for the reduction of assembly costs). Meanwhile, the module that executes function 5 requires 3 variations to generate the variety of supply desired.

The architectural alternative of modular focus suggested by the application of the metric proposed for the family of products used as an example with $\alpha$ equal to 0.3 and $\beta$ equal to 0.7 result in:

- total estimated costs, relative to the other architectures, of the alternatives suggested and illustrated in Figure 9 of 0.78141 , the lowest of the 52 possible alternative architectures of modular focus for products that execute 5 functions (the estimated cost is composed, as proposed, of the development and assembly costs);

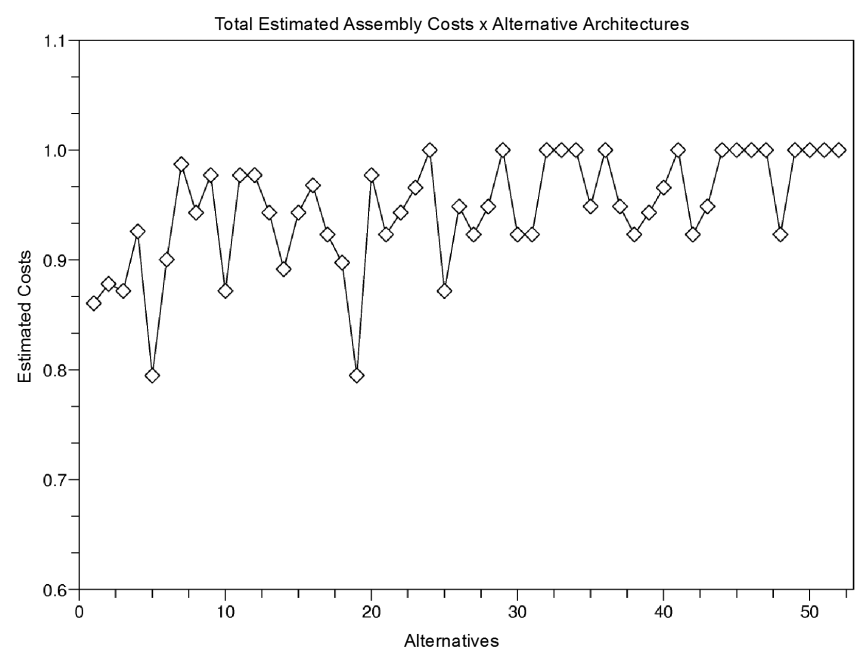

Figure 7. Estimate of the total assembly cost for the various architectural alternatives.

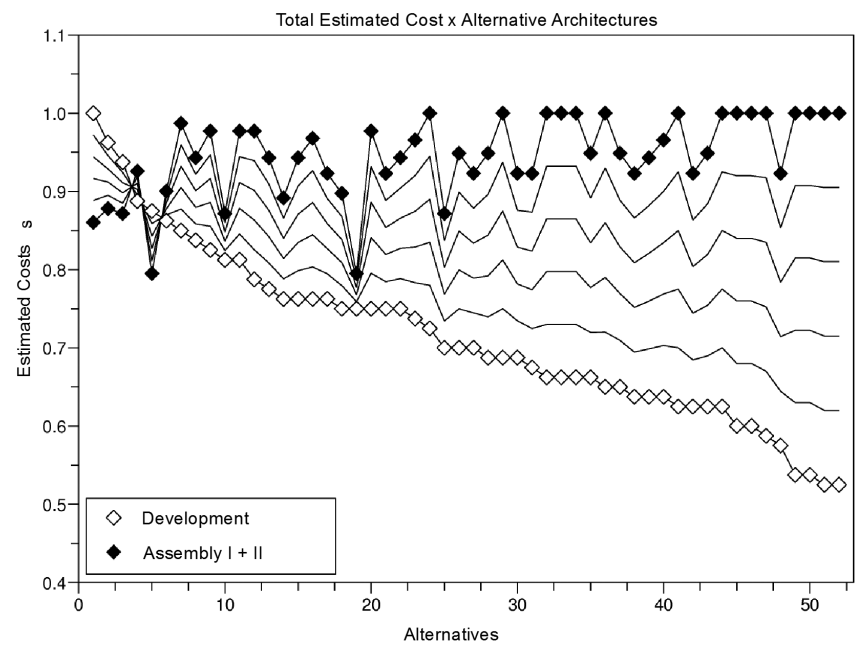

Figure 8. Variation in total cost as a function of $\alpha$ and $\beta$ for the alternative architectures.
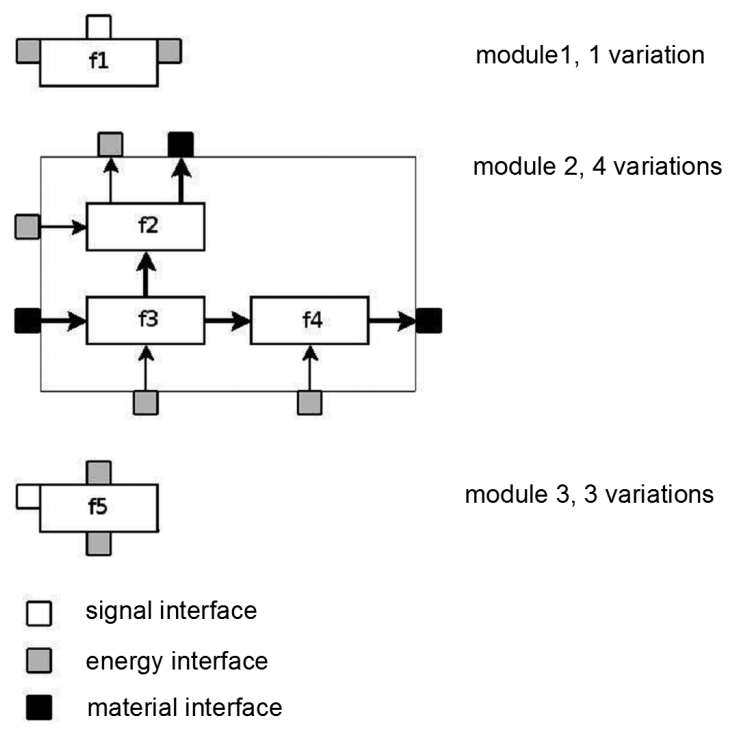

module 3,3 variations

Figure 9. Modules suggested for the product family. 
- the development cost of this alternative architecture for the family of products is $75 \%$ of what would be the development costs of the four products according to a totally integral architecture;

- estimated assembly cost of the inter-module interfaces for the architecture suggested, $69 \%$ of what would be the assembly costs of these products according to a totally modular architecture.

- Estimated assembly costs for the inter-module interfaces (only in the case of module 2) $10 \%$ of what would be the assembly costs of the products according to, once again, a totally modular architecture.

\section{Conclusion}

The metric proposed in this article meets the objective of making relative evaluations of the alternative architectures for the product family. The results expected from the application of this metric to the simplified functional models of the products were confirmed. The use of a measure, such as that proposed in this article, for the evaluation and definition of the architecture of a product family makes the process of modularization independent of the familiarity of the designers with this development concept. Its adoption is an alternative solution to the important problem of definition of an architecture for products, establishing an order for the most attractive alternatives. For simple products, or that is those with few functions, the application of the metric is direct, as was shown in the example developed in this article. Nevertheless, the alternative architectures for the products grew considerably as the number of functions increased, making nonviable the study of all the alternative architectures as shown in the example given in the article. These cases require the assistance of computing tools to establish an order for the alternatives. Fortunately, with the increased processing capacity of computers at low costs, approaches to the search for the best architectural alternatives for products assisted by computing tools is at everyone's reach. The computing tools that are suitable to this investigation include the evolutionary algorithms. Genetic Algorithms in particular demonstrate great potential in assisting the definition of the product architecture. Preliminary results obtained by the authors indicate that Genetic Algorithms are suitable to the study of the space of alternative architecture for products. Nevertheless, although promising, the use of this computing tool in the assistance of the choice of architecture of products is still being investigated.

\section{References}

BROWNING, T. R. Applying the design structure matrix to system decomposition and integration problems: a review and new directions. IEEE Transactions on Engineering Management, v. 48, n. 3, p. 292-306, 2001. http://dx.doi. org/10.1109/17.946528.

DAHMUS, J. B.; GONZALEZ-ZUGASTI, J. P.; OTTO, K. N. Modular product architecture. Design Studies, v. 22, n. 5, p. 409-424, 2001. http://dx.doi.org/10.1016/S0142694X(01)00004-7.

DICKAU, R. M. Mathematical figures: using mathematica. 2006. Available from: <http://mathforum.org/advanced/ robertd/index.html>. Access in: 11 Mês 2006.

ERENS, F.; VERHULST, K. Architectures for product families. Computers in Industry, v. 33, n. 2-3, p. 165-178, 1997. http://dx.doi.org/10.1016/S0166-3615(97)00022-5.

ERIXON, G. Modular Function Deployment (MFD), support for good product structure creation. In: WDK WORKSHOP ON PRODUCT STRUTURING, 2., 2006, Netherlands. Proceedings... Netherlands: Delft University of Technology, 1996.

HÖLTTÄ-OTTO, K. Modular product platform design. 2005. Dissertação (Mestrado em Design de Máquinas)Departament of Mechanical Engineering, Helsinki University of Technology, Espoo, 2005.

HUANG, C.-C.; KUSIAK, A. Modularity in design of product systems. IEEE Transactions on Systems, v. 28, n. 1, p. 66-77, 1998. http://dx.doi.org/10.1109/3468.650323.

PAHL, G. et al. Projeto na engenharia. São Paulo: Edgard Blücher, 2005.

STONE, R. B.; WOOD, K. L.; CRAWFORD, R. H. A heuristic method to identify modules from a functional description of a product. In: DESIGN ENGINEERING TECHNICALDETC98, 1998, Atlanta, Georgia. Proceedings... Atlanta, Georgia: DETC98, 1998.

ULRICH, K. The role of product architecture in the manufacturing firm. Research Policy, v. 24, n. 3, p. 419-440, 1995. http://dx.doi.org/10.1016/0048-7333(94)00775-3.

YANG, T. G.; BEITER, K. A.; ISHII, K. Product platform development: considering product maturity and morphology. In: INTERNATIONAL MECHANICAL ENGINEERING CONGRESS AND EXPOSITION-IMECE, 2005, Orlando, Florida, USA. Proceedings... Orlando, Florida, USA: IMECE, 2005. p. 425-435. http://dx.doi.org/10.1115/ IMECE2005-82886. 